\title{
Parallel Implementation for Probabilistic Analysis of 3D Discrete Cracking in Concrete
}

\author{
Camen N. M. Paz ${ }^{1}$, Luiz F. Martha ${ }^{1}$, José L.D. Alves, ${ }^{2}$ Eduardo, M.R. Fairbairn ${ }^{2}$, \\ Nelson F.F. Ebecken ${ }^{2}$ and Alvaro L.G.A. Coutinho ${ }^{2}$ \\ ${ }^{1}$ Department of Civil Engineering \\ Pontifical Catholic University of Rio de Janeiro, (PUC-Rio) \\ Street Marquês de São Vicente 225, 22453-900 - Brazil \\ $\{$ mena,lfm\}@tecgraf.puc-rio.br \\ ${ }^{2} \mathrm{COPPE} / \mathrm{UFRJ}$ - Program of Civil Engineering \\ PO Box 68506, 21945-970 \\ Federal University of Rio de Janeiro - Brazil \\ jalves@1amce.ufrj.br, eduardo@labest.coc.ufrj.br, nelson@ntt.ufrj.br, alvaro@nacad.ufrj.br
}

\begin{abstract}
This work presents computational strategies used in an implementation of the probabilistic discrete cracking model for concrete of Rossi suitable to parallel vector processor (PVP). The computational strategies used for each realization, within the framework of Monte Carlo simulation, are the inexact Newton method to solve the highly nonlinear problem and element-by-element (EBE) iterative strategies considering that nonlinear behavior is restricted to interface elements. The simulation of a direct tension test is used to illustrate the influence of adaptive inexact Newton strategy in code performance on a CRAY T90
\end{abstract}

\section{Introduction}

Concrete, the most used construction material in the world, is a very heterogeneous material, because of its composite structure composed by aggregates, mortar, fibers, etc. and also because of the physical phenomena that take place during chemical and physical evolution: autogenous shrinkage, initial stresses and microcracking, etc. The material can also present fracture size effects that may introduce large differences for the ultimate loads and non-objectivity of the analysis. To handle with these characteristics, two basic approaches are generally used to model the mechanical behavior of the material: (i) the heterogeneous material is assimilated to a statistically equivalent homogeneous material and a deterministic approach together with a size effect law is used; (ii) homogeneity is no longer considered, at a certain scale, and the heterogeneity of the material, introduced by a stochastic approach, is the responsible for the scale effect.

Regarding concrete, the second approach is quite recent, and in this paper we refer to Rossi's et al. [1], [2], [3], [4] probabilistic model that seems to take into account size effects when the statistical parameters are well determined. This method uses Monte Carlo simulations to find the average behavior of the structure, which corresponds to the converged mean values of some typical results. 
The remaining of this work is organized as follows; section 2 describes the probabilistic model; section 3 describes the formulation of the interface element; section 4 describes the computational strategies employed and section 5 addresses the numerical experiments carried out. Finally, section 6 closes this work presenting some conclusions drawn from the experiments.

\section{Probabilistic Model}

Among several other relevant factors, such as water/cement ratio of the paste, casting and curing conditions, loading conditions, etc, concrete cracking depends on the random distribution of constituents and initial defects. The heterogeneity governs the overall cracking behavior and related size effects on concrete fracture. The probabilistic crack approach, based on the direct Monte Carlo method, developed by Rossi and co-workers ( Rossi et al. [1], [2], [3], [4] ) takes this stochastic process into account by assigning in finite element analysis, randomly distributed material properties, such as tensile strength and Young's modulus to both the solid elements and the contact elements interfacing the former, that is, a discrete crack approach. This approach considers that all nonlinearities are restricted to contact elements modeling cracks. Therefore, the stochastic process is introduced at the local scale of the material, by considering that cracks are created within the concrete with different energy dissipation depending on the spatial distribution of constituents and initial defects. The local behavior is assumed to obey a perfect elastic brittle material law. Thus, the random distribution of local cracking energies can be replaced by a random distribution of local strengths. The present probabilistic model involves a number of mechanical properties of the material to be determined, which constitutes the modeling data. From a large number of direct tensile tests, it was found that a normal law describes rather well the experimental distribution of the relevant material data (Rossi et al. [2]). These characteristics are the means of the tensile strength $\left(f_{c t, \mu}\right)$ and of the Young's modulus $\left(E_{\mu}\right)$; the standard deviations of the tensile strength $\left(f_{c t, \sigma}\right)$ and of the Young's modulus $\left(E_{\sigma}\right)$. The following analytical expressions were proposed (Rossi et al. [2]):

$$
\begin{gathered}
f_{c t, \mu}=6.5 \cdot C \cdot\left(V_{T} / V_{g}\right)^{-a} \\
E_{\mu}=E \\
E_{\sigma} / E=0.15\left(V_{t} / V_{g}\right)^{-c} \\
a=0.25-3.6 \cdot 10^{-3}\left(f_{c} / C\right)+1.3 \cdot 10^{-5}\left(f_{c} / C\right)^{2} \\
b=4.5 \cdot 10^{-2}+4.5 \cdot 10^{-3}\left(f_{c} / C\right)+1.8 \cdot 10^{-5}\left(f_{c} / C\right)^{2} \\
c=0.116+2.7 \cdot 10^{-3}\left(f_{c} / C\right)-3.4 \cdot 10^{-6}\left(f_{c} / C\right)^{2}
\end{gathered}
$$

where $V_{T}$ is the volume of the two finite elements contiguous to an individual contact element of the mesh, $V_{g}$ is the volume of the coarsest grain, $C=1 M P a, f_{c}$ is the cylinder compressive strength determined by the standardized test (French standard) on a cylinder $160 \mathrm{~mm}$ in diameter and $320 \mathrm{~mm}$ high, and $E$ is the mean value (obtained from tests) of the Young's modulus which does not exhibit significant volume effects. 
In these expressions, the compressive strength $f_{c}$ represents the quality of the concrete matrix, while the volume of the coarsest aggregate $V_{g}$, refers to the elementary material heterogeneity.

Equations (1) to (3) show that the smaller the scale of observation, the larger the fluctuation of the local mechanical properties, and thus the (modeled) heterogeneity of the matter. In other words, the finer the mesh, the greater the modeled heterogeneity in terms of Young's modulus and tensile strength. The empirical expressions corresponding to equations (1) to (3) were calibrated to fit a number of material tests for different types of concretes and volumes. They give a first approximation to capture volume effects on concrete fracture, but they are not expected to be universal. Furthermore, little is known about the validity of these empirical formulas for small $V_{t} / V_{g}$. These volume ratios, which may be used in finite element structural analysis, are too little to be determined by means of experimental tests.

The mesh has $n v$ volume elements and $n i$ interface elements. Each interface element follows a rigid-brittle constitutive law characterized by an individual tensile strength $f_{c t, i}$.

The volume elements are elastic and have individual Young's modulus referenced by $E_{v}$.

Following Rossi et al. [2] findings, these individual local tensile strengths and Young's modulus are represented by normal distributions having the densities:

$$
\begin{aligned}
& g_{f}\left(f_{c t}\right)=\frac{1}{f_{c t, \sigma} \sqrt{2 \pi}} \exp \left[-\frac{1}{2}\left(\frac{f_{c t, \mu}}{f_{c t, \sigma}}\right)^{2}\right] \\
& g_{E}(E)=\frac{1}{E_{\sigma} \sqrt{2 \pi}} \exp \left[-\frac{1}{2}\left(\frac{E-E_{\mu}}{E_{\sigma}}\right)^{2}\right]
\end{aligned}
$$

where $g_{f}\left(f_{c t}\right)$ and $g_{E}(E)$ are the density function for the tensile strength $f_{c t}$ and the Young's modulus $E, x_{\mu}$ and $x_{\sigma}$ denote the mean and standard derivation of the distribution of quantity $x$. For the problem at hand, it is possible to find a sample of $n i$ values $f_{c t, i}$, each value corresponding to an interface element, and $n v$ values $E_{v}$, each value corresponding to a volume element, by using a standard routine for generation of random numbers for a given normal distribution (Press et al., [6]).

If the heterogeneous characteristics of the material are well established and quantified by the statistical moments it is possible that the model displays the size effects related to the material heterogeneity. The problem with this approach is that these statistical moments are not known, a priori, for the characteristic volume of the finite elements used. However, some methods have been proposed to determine these parameters by means of inverse analysis using neural networks (Fairbairn et al, [7],[8] ).

The solution for this probabilistic approach is obtained by means of a Monte Carlo simulation. A number of $n$ samples is generated for a given normal distribution, and some characteristic responses of the structure are computed; for example, stress crack-width $\sigma-w$ curve, or load displacement $P-\delta$ curve. Let the $j$ th samples correspond to, e.g., the $j$ th $\sigma-w$ curve. This $j$ th $\sigma-w$ curve is composed of discrete values, $\sigma_{k}^{j}$ and $w_{k}^{j}$, where 
the superscript $j$ indicates the sample and the subscript $k$ the discrete value of the $\sigma-w$ curve. The same discrete $w$-values are assumed and the response is defined exclusively by the values of $\sigma_{k}^{j}$. The mean curve composed by pairs $\sigma_{k}^{\text {mean }}, w_{k}$ then simply reads

$$
\sigma_{k}^{\text {mean }, j}=\frac{1}{j} \sum_{l=1}^{j} \sigma_{k}^{l}
$$

The Monte Carlo simulation is stopped when

$$
\left|\sigma_{k}^{\text {mean }, j}-\sigma_{k}^{\text {mean }, j-1}\right| \leq \text { tol }
$$

where $t o l$ is the prescribed tolerance to check the convergence of the procedure. With the convergence of the procedure, the total number of samples is set to $n=j$. This total number of samples $n$, corresponding to a Monte Carlo converged simulation, clearly depends on tol, which is a measure of the precision required by the analysis. It also depends on the heterogeneity of the material represented by the standard deviation. The more heterogeneous is the material the greater is the number of samples necessary to obtain a converged solution. Our experience in this field indicates that 25 to 50 samples are sufficient to obtain a converged $\sigma-w$ curve.

\section{Interface elements for modeling discrete cracking}

The finite element cracking model is a discrete model for which volume elements are always elastic and cracking occurs in elastic-brittle (almost rigid brittle) contact elements placed between two volume elements. The three dimensions interface element (Paz, [5]) depicted in figure 1 can be thought as a triangular base prism connecting adjacent faces of neighboring tetrahedra. These elements are formulated to describe relative displacements between the triangular faces simulating crack opening.

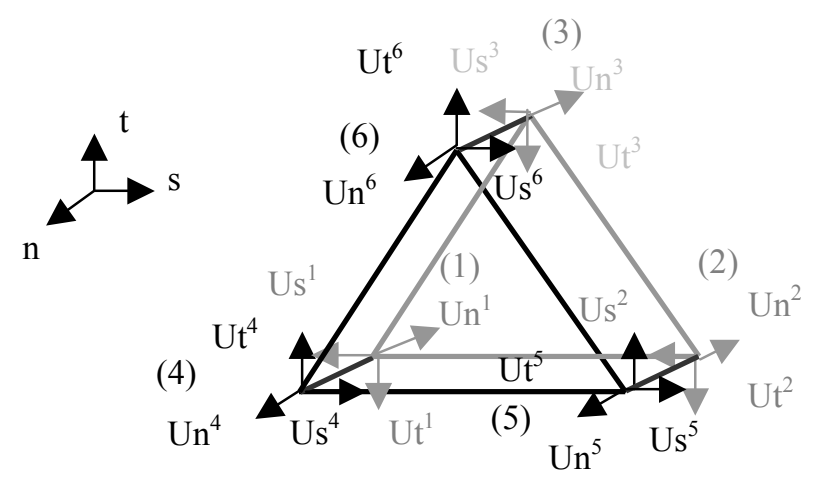

Fig. 1. An Interface Element and its degrees of freedom in a local system

The constituve law of the 3D interface element is defined by equation (8) for non cracked elastic state characterized by $\sigma_{n}<f_{c t, i}$. When the tensile strength is reached the elements attains the cracked stage and modulus $E_{c}$ and $G_{c}$ are set to zero (figure 2). 


$$
\Delta \boldsymbol{\sigma}=\mathbf{D}_{c r} \Delta \mathbf{w}=\left\{\begin{array}{l}
\Delta \sigma_{n} \\
\Delta \sigma_{s} \\
\Delta \sigma_{t}
\end{array}\right\}=\left[\begin{array}{ccc}
E_{c} / h & 0 & 0 \\
0 & G_{c} / h & 0 \\
0 & 0 & G_{c} / h
\end{array}\right]\left\{\begin{array}{l}
\Delta w_{n} \\
\Delta w_{s} \\
\Delta w_{t}
\end{array}\right\}
$$

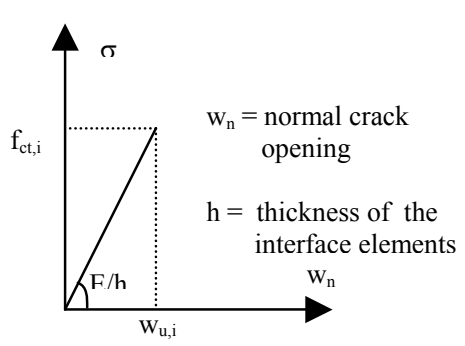

Fig. 2. Elastic-Brittle Contact law

In equation (8) the subscript $n$ normal stands for, while stand for $s$ and $t$ tangential indicating the direction respective to the crack plane, $w$ are the relative displacements between the two faces of the interface element, $h$ is the width of interface element, $E_{c}$ and $G_{c}$ are respectively the normal (Young's) and the shear modulus along crack plane.

Equations (8) and figure 2 define the elastic-britle constituve behavior. The thickness $h$ plays the role of a penalization parameters and should be conveniently chosen not to affect the solution.

The kinematic relation for the interface element is;

$$
\Delta \mathbf{w}=\mathbf{B} \Delta \mathbf{a}_{l}^{e}
$$

where

$$
\mathbf{B}=\frac{1}{3}\left[\begin{array}{cccccccccccccccccc}
-1 & 0 & 0 & -1 & 0 & 0 & -1 & 0 & 0 & 1 & 0 & 0 & 1 & 0 & 0 & 1 & 0 & 0 \\
0 & -1 & 0 & 0 & -1 & 0 & 0 & -1 & 0 & 0 & 1 & 0 & 0 & 1 & 0 & 0 & 1 & 0 \\
0 & 0 & -1 & 0 & 0 & -1 & 0 & 0 & -1 & 0 & 0 & 1 & 0 & 0 & 1 & 0 & 0 & 1
\end{array}\right]
$$

$\Delta \mathbf{w}$ is the crack opening incremental and $\Delta \mathbf{a}_{1}^{\mathrm{e}}$ is the vector incremental nodal displacements for the interface element

Applying a standard displacement based F. E. formulation, the resulting tangent stiffness matrix for the interface element is given is:

$$
\mathbf{K}_{\text {Intf }}^{\mathrm{e}}=\int_{\Omega} \mathbf{B}^{T} \mathbf{D}_{c r} \mathbf{B} \mathrm{d} \Omega
$$

The interface elements are generated contiguous to the faces of selected tetrahedra elements. This selection is performed by the user, defining a 3D box inside the mesh that contains the target elements. 
Remark Our experience indicates that to increase robustness of nonlinear solution process we have to limit only one interface element to "crack" at each nonlinear iteration.

\section{Computational strategies.}

In this work we employ the inexact Newton method (Kelley, [9]) to solve resulting nonlinear set of equations at each load or displacement increment.

The outline of the nonlinear solution algorithm is presented in Box 1.

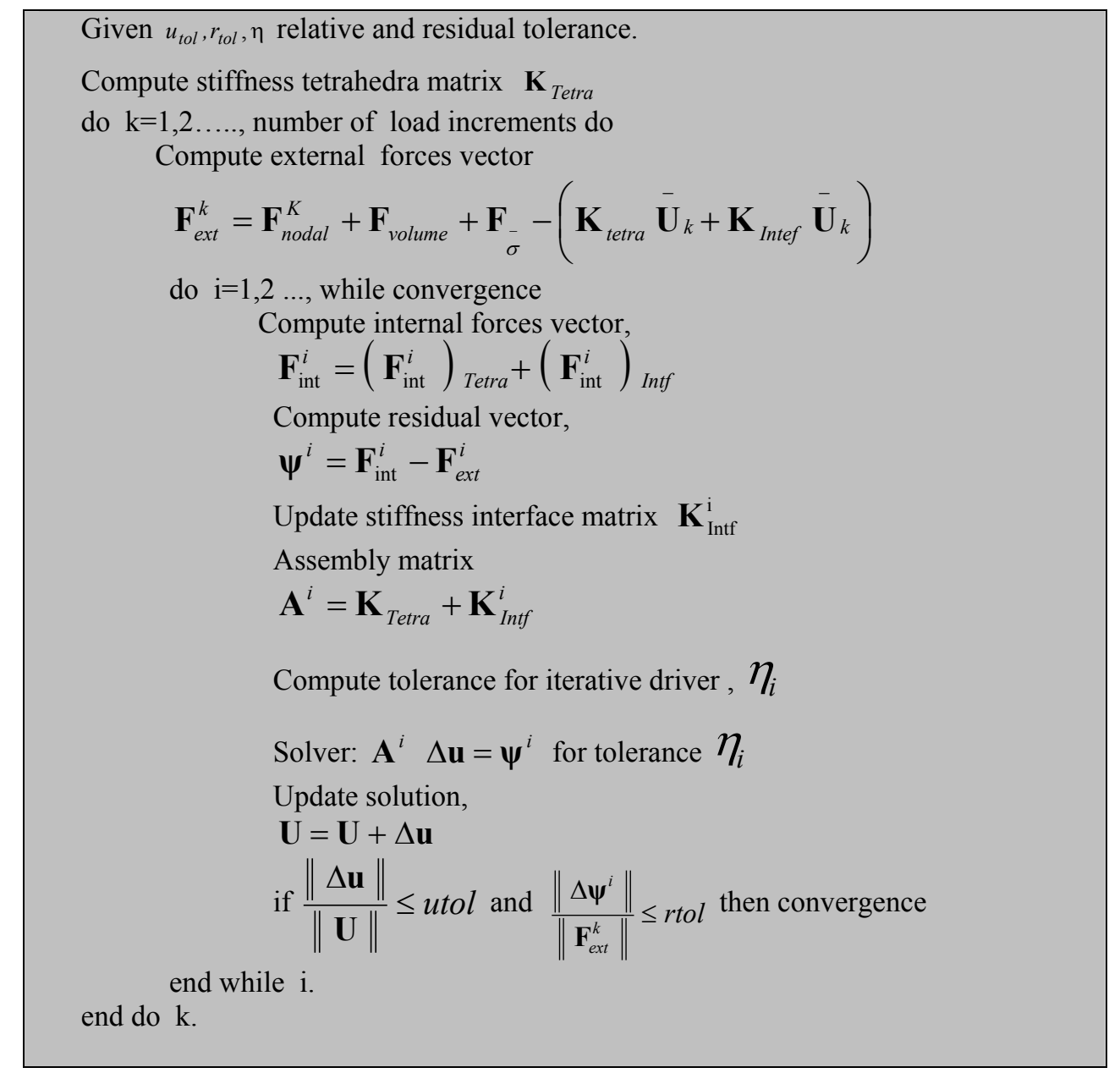

Box 1. Inexact Newton Algorithm

Note that in $\mathbf{F}_{\text {ext }}^{k}$ we account for nodal forces, body forces and prescribed displacements and stresses $\overline{\mathbf{U}}, \overline{\boldsymbol{\sigma}}$. The total internal forces vector $\mathbf{F}_{\text {int }}^{i}$ is the sum of the tetrahedra element vector internal forces $\left(\mathbf{F}_{\text {int }}^{i}\right)_{\text {Tetra }}$ plus the interface element internal forces vector $\left(\mathbf{F}_{\text {int }}^{i}\right)_{\text {Intf }}$. The total stiffness matrix is the sum of the continuum matrix $\mathbf{K}_{\text {Tetra }}$ plus the 
interface matrix $\mathbf{K}_{\text {Intf }}^{i}$ updated at every nonlinear iteration. An approximate solution is obtained when the Inexact Newton termination criterion is satisfied, that is

$$
\left\|\mathbf{A}^{i} \Delta \mathbf{u}-\boldsymbol{\psi}^{i}\right\| \leq \eta_{i}\left\|\boldsymbol{\psi}^{i}\right\|
$$

Tolerance $\eta_{\mathrm{i}}$ may be kept fixed throughout the nonlinear iterations or may be adaptively selected as the iterations progress towards the solution. Our choice for $\eta_{i}$ follows the criteria suggested by Kelley.

The iterative driver for the solution of the linearized set of equations is the element-byelement PCG. Several preconditioner options are available ranging from the simple diagonal up to incomplete factorizations. In this work, to stress the benefits of the inexact Newton method, we restricted ourselves to simple diagonal and nodal block diagonal.

\section{Remarks}

1) In the present implementation matrix vector products in EBE, PCG are computed as:

$$
\mathbf{A} \mathbf{p}=\sum_{\mathrm{i}=1}^{\mathrm{N}_{\text {tetra }}}\left(\begin{array}{ll}
\mathbf{K}_{\text {Tetra }} & \mathbf{p}_{\mathrm{i}}
\end{array}\right)+\sum_{\mathrm{j}=1}^{\mathrm{N}_{\text {Intf }}}\left(\begin{array}{ll}
\mathbf{K}_{\text {Intf }} & \mathbf{p}_{\mathrm{j}}
\end{array}\right)
$$

where, $\mathrm{N}_{\text {tetra }}$ is the number of tetrahedra, $\mathrm{N}_{\text {intf }}$ is the number of interface elements, $\mathbf{K}_{\text {Tetra }}$ and $\mathbf{K}_{\text {Intf }}$ are respectively element matrices for the tetrahedra and interface; $\mathbf{p}_{\mathrm{i}}$ and $\mathbf{p}_{\mathrm{j}}$ are the components of $\mathbf{p}$ restricted to the degrees of freedom of two element type.

2) Stiffness matrices for tetrahedra are computed and stored at the beginning of the analysis since they are elastic.

3) Stiffness matrix for interface elements are updated at every nonlinear iteration.

4) The arrays of element stiffness matrices are stored taking into account their symmetry; in the case of the element tetrahedra 78 coefficients are stored and for the interface element only 18 coefficients are stored, exploring the particular structure of the discrete gradient operator given in equation (10).

5) The mesh coloring algorithm of Hughes [10] was extended to block both solid and interface elements into disjoint groups thus enabling full vectorization an parallelization of the operations involved in equation (13).

\section{Numerical experiments}

\subsection{Direct tension test}

The experimental results of concrete uniaxial tension tests published by (Li et al [12] ) were used to validate the developments presented in this paper. The specimens are cylinders $101.6 \mathrm{~mm}$ in diameter and $203.2 \mathrm{~mm}$ high. This specimens had $25.4 \mathrm{~mm}$ notches at their midheight on both sides (figure 3 ).

Concrete with maximum aggregate diameter of $9.525 \mathrm{~mm}$ was used. Its average tensile strength and Young's modulus at the age of 28 days were: $f_{c t}=4,72 \quad M P a$ and $E_{c}=42000 \mathrm{MPa}$. 


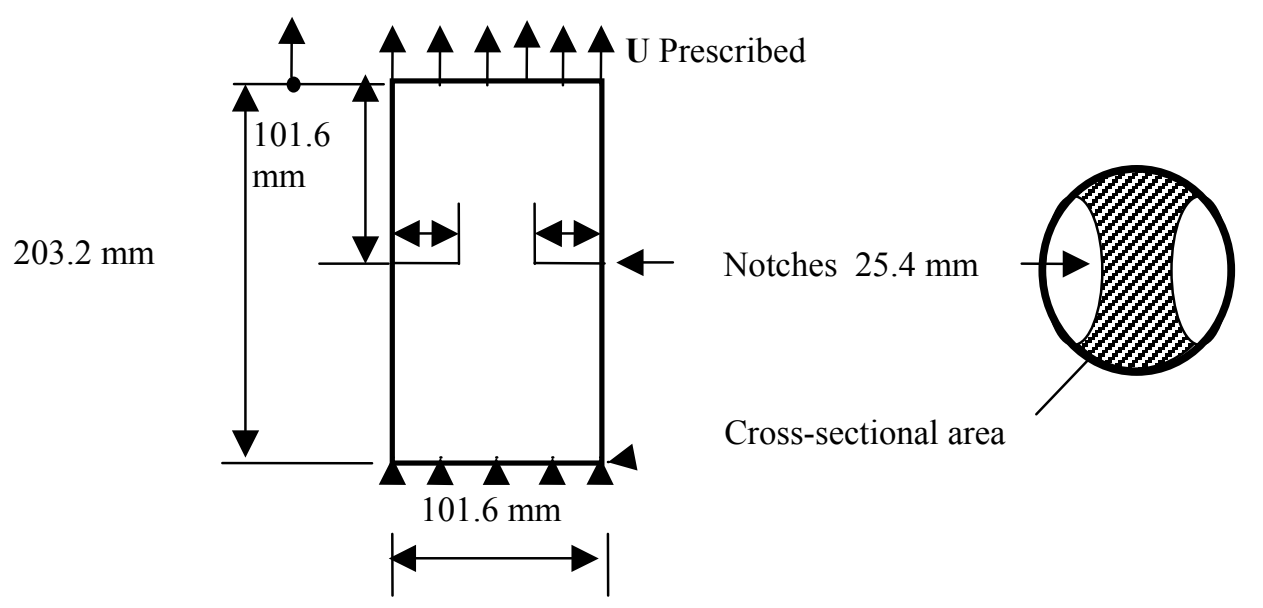

Fig. 3. Uniaxial tension specimen geometry, dimensions, load, boundary conditions

The numerical experiments were controlled by a field of uniform displacements applied at the top of the test specimen in 30 incremental steps $\Delta u=5.3 \times 10^{-6}$. The boundary conditions restrain the degrees of freedom in the vertical at the bottom. The final mesh has 11,933 elements, where 4,775 are tetrahedra and 7,158 are interface elements (figure 4).

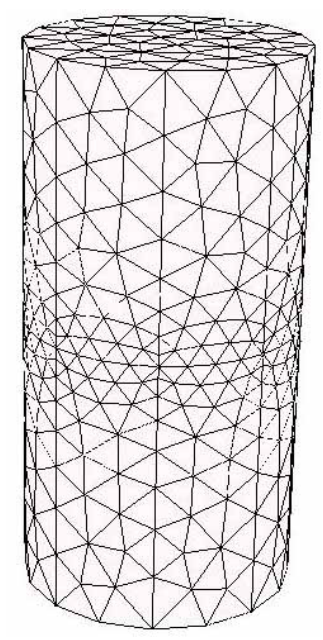

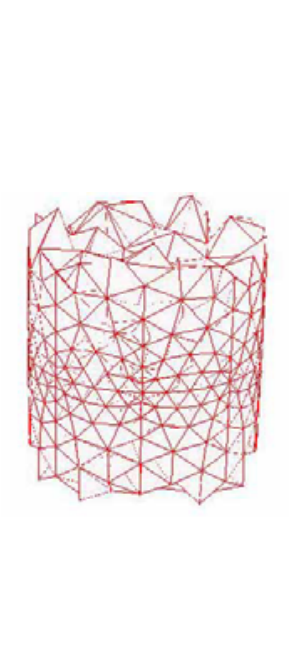

(a)

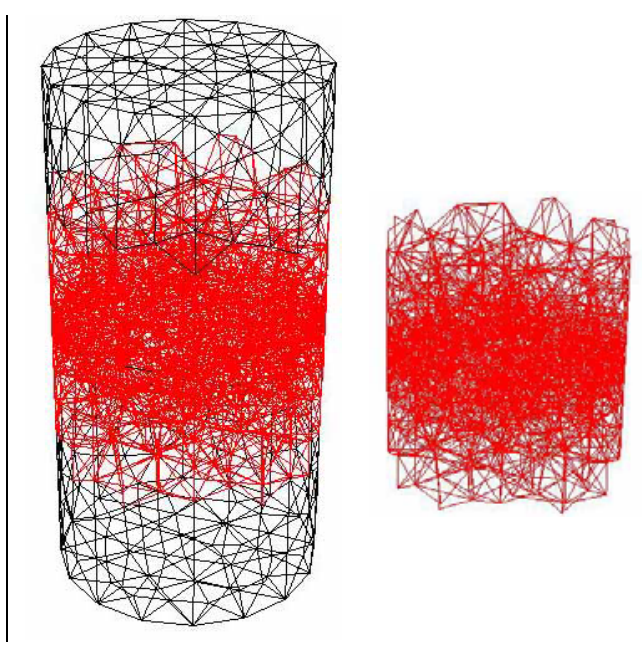

(b)

Fig. 4. Hidden line (a) and wire frame (b) representations of the computational mesh for the simulation of a direct tension test. It is also shown aside the resulting mesh for the interface elements considering. 


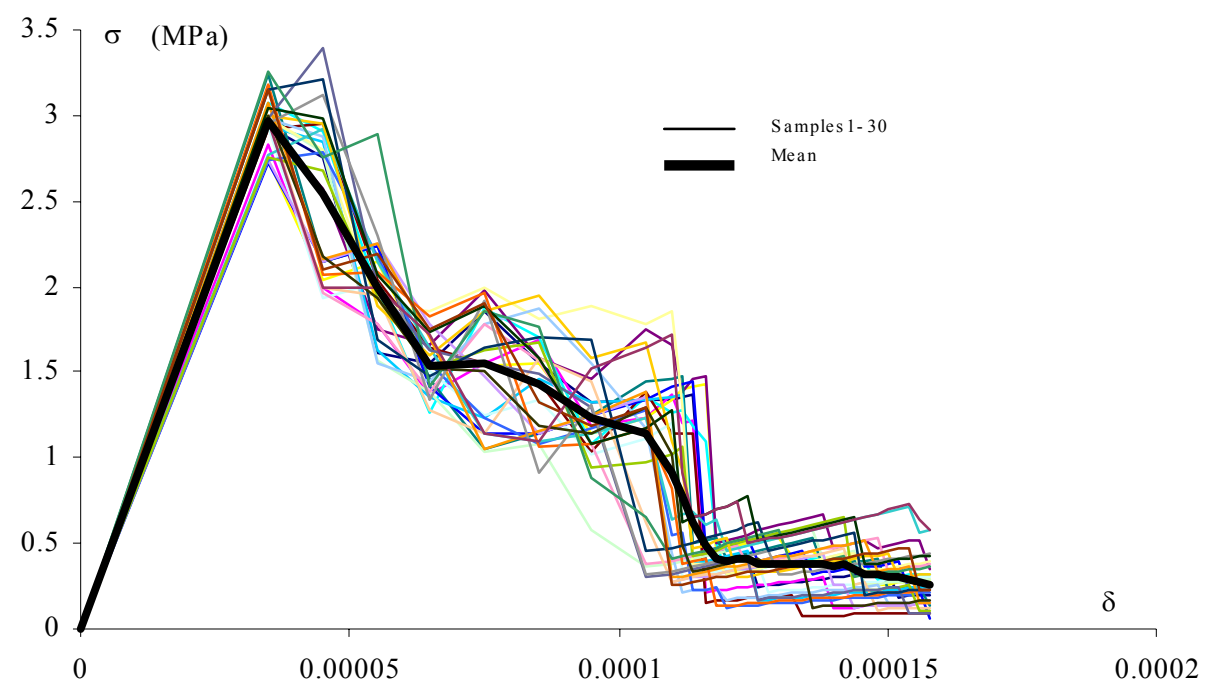

Fig. 5. Results for the complete Monte Carlo simulation.

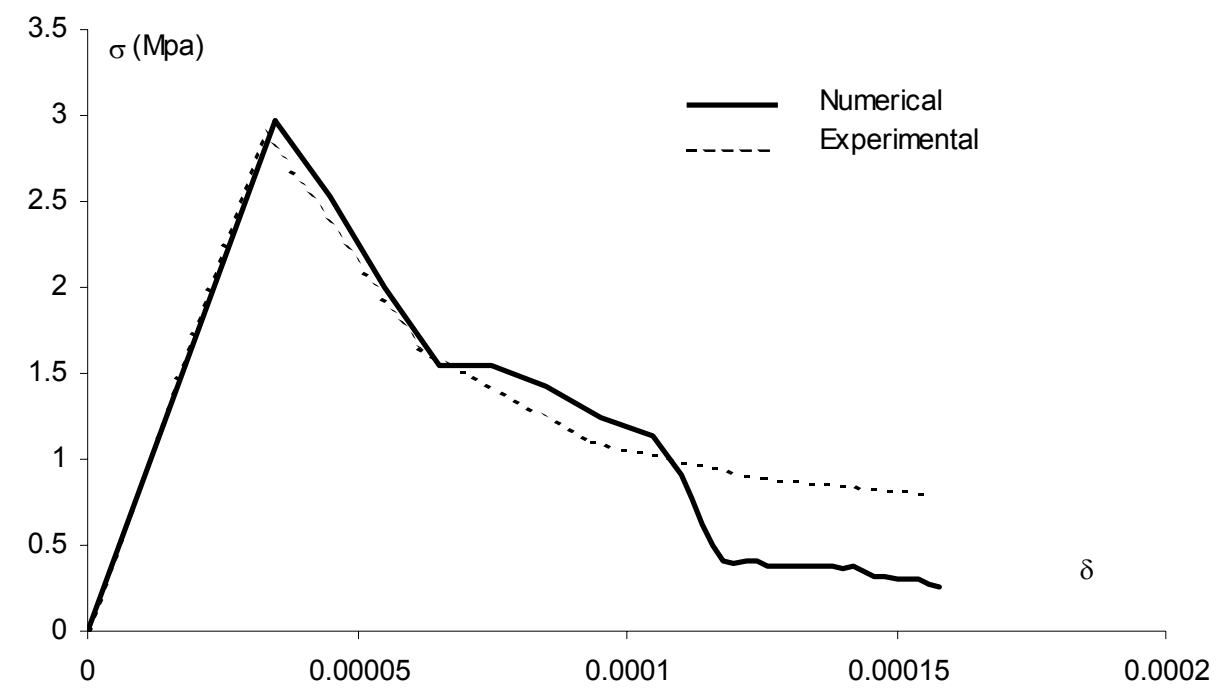

Fig.6. Comparison of experimental and numerical results. 
Curves $\sigma-\delta$ for the several realizations of the Monte-Carlo simulation are given in figure 5. The comparison between experimental by Li et al [12] and the convergent Monte Carlo $\sigma-\delta$ curve is given in figure 6 , figure 7 presents the crack configuration for a given sample at a stage corresponding to the softening branch of the $\sigma-\delta$ curve
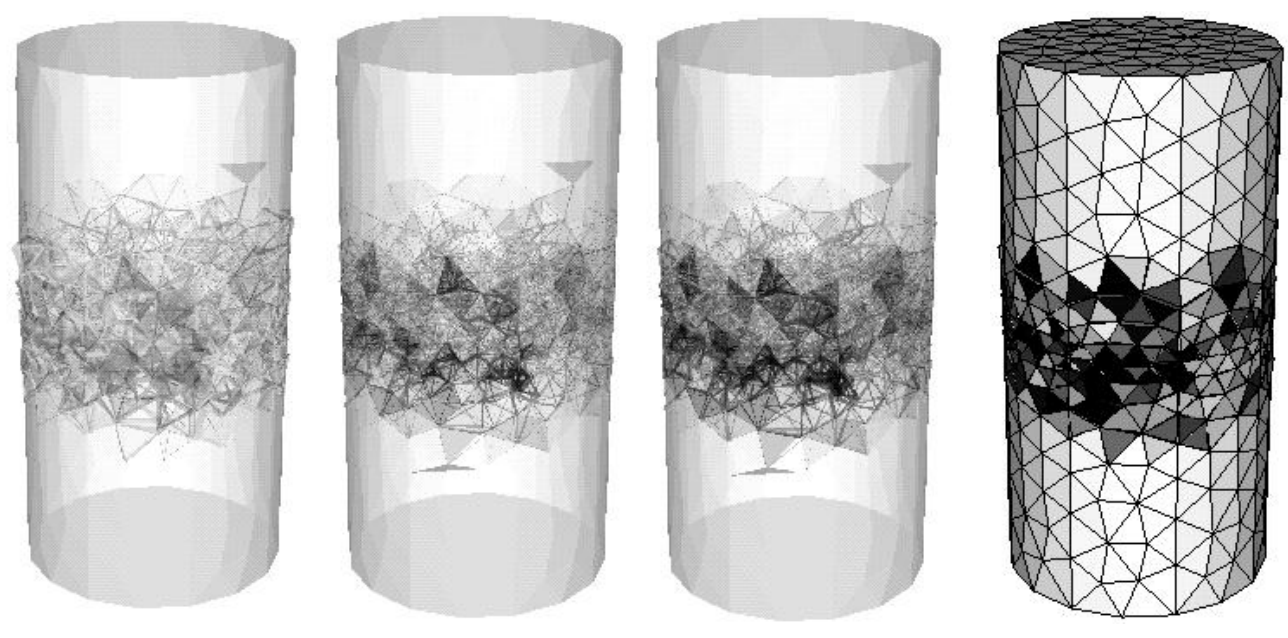

Fig. 7. Crack evolution for numerical simulation

\subsection{Comparison of inexact Newton strategies}

An assessment of the implemented inexact Newton strategies was carried out for one realization of the direct tension test simulation the selected preconditioner for this experiment was the diagonal nodal block.

A fixed tolerance $\eta=10^{-3}$ for PCG was selected for the first analysis requiring 52 inexact Newton iterations. For the second analysis, now employing the adaptive inexact Newton method, the tolerance range for PCG was set to $10^{-3} \leq \eta \leq 10^{-6}$, requiring 54 inexact Newton iterations. Tolerances for the inexact Newton were iterations set to $\mathrm{u}_{\mathrm{tol}}=\mathrm{r}_{\mathrm{tol}}=10^{-3}$, in both cases.

As can be seen in Figure (8), comparing the number of nonlinear iterations for both analyses, it remained fairly the same irrespective to the selection of the inexact Newton approach, either fixed or adaptive, indicating that the overall nonlinear character of the solution was not affected the adaptive approach. Figure (9) shows a plot of the number of PCG iterations per incremental step for both analyses. It is evident in this figure the reduction of PCG iterations due to the adaptive strategy. 


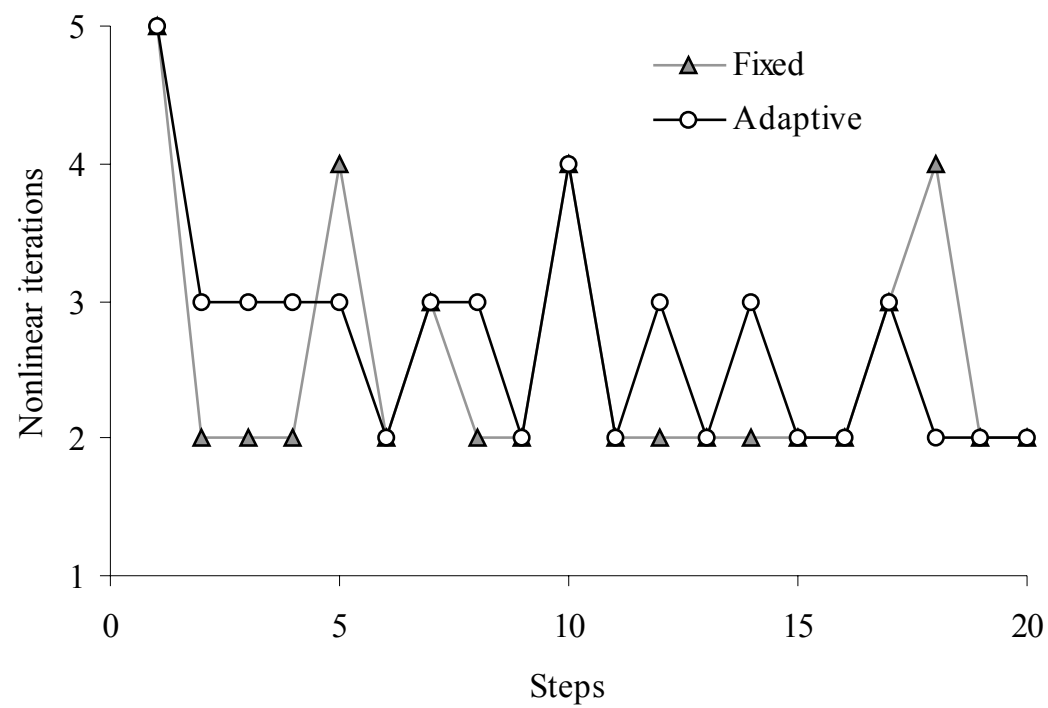

Fig. 8. Nonlinear iterations per incremental step for the direct tension test simulation.

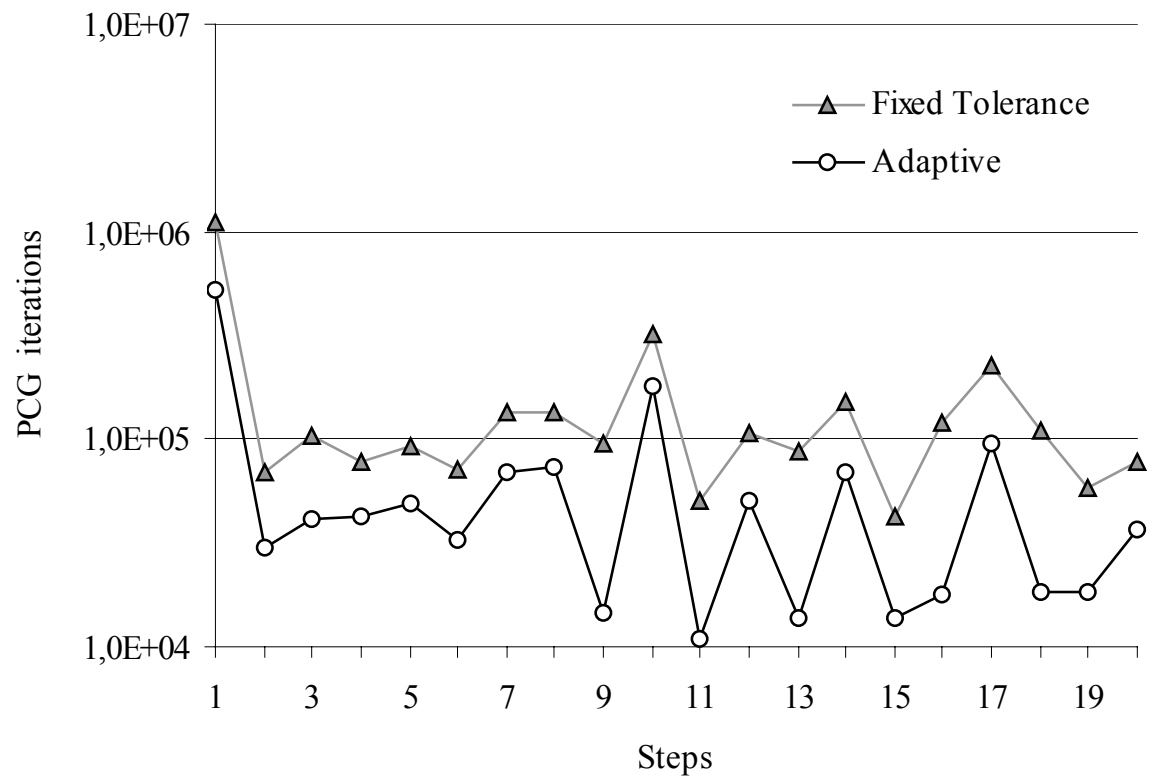

Fig. 9. PCG iterations per incremental step for the direct tension test simulation.

\subsection{Computational Performance}

A detailed vector performance analysis is obtained by the summary of the PERFVIEW's Report presented in Table 3. The CPU time of the vectorized single processor run for 
CRAY T90 is 10.38 hours. This table list for a single CPU run, the Mflop/s rates for the three top routines. The routines Smatv-fint and Smatv-tetra are respectively responsible for the matrix-vector products for the interface and tetrahedra elements, routines are the computations kernels the routine PCG-block, implements the iterative solver the nodal block diagonal Preconditioned Conjugate Gradient.

\begin{tabular}{|c|c|c|}
\hline Routines & Single CPU (\%) & Performance (Mflop/s) \\
\hline smatv-intf & 52.80 & 613.7 \\
\hline smatv-tetra & 17.32 & 554.0 \\
\hline PCG-block & 25.90 & 82.4 \\
\hline Others & 3.98 & - \\
\hline
\end{tabular}

Table 3 Performance Analysis -The top 3 subroutines

The code achieved good vectorization on the CRAY T90 for a mesh with 11933 elements, comprising (7158 interface and 4775 tetrahedra elements). The top three subroutines consume the major CPU utilization in the whole analysis.

The parallel performance is shown in table 4 and the figure 10 (a) and (b) as obtained from a summary ATEXPERT report, autotasking performance tool. The routines Fint-tetra and Fint-intf evaluate respectively the internal force vector of the interface elements and the tetrahedra elements. Routine A-Kintf computer update interface stiffness..

\begin{tabular}{|c|c|c|c|}
\hline Routines & \% Parallel & $\begin{array}{c}\text { Dedicated } \\
\text { Speedup }\end{array}$ & Actual Speedup \\
\hline Smatv-tetra & 98.9 & 3.96 & 3.8 \\
\hline Smatv-intf & 99.8 & 3.83 & 3.8 \\
\hline Fint-tetra & 92.5 & 3.83 & 3.2 \\
\hline Fint-intf & 99.1 & 3.55 & 3.5 \\
\hline A-Kintf & 85.8 & 3.53 & 2.6 \\
\hline
\end{tabular}

Table 4 Summary of the ATEXPERT's Report for the 5 dominant loops

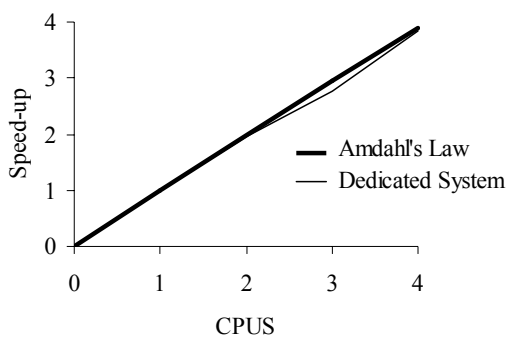

(a)

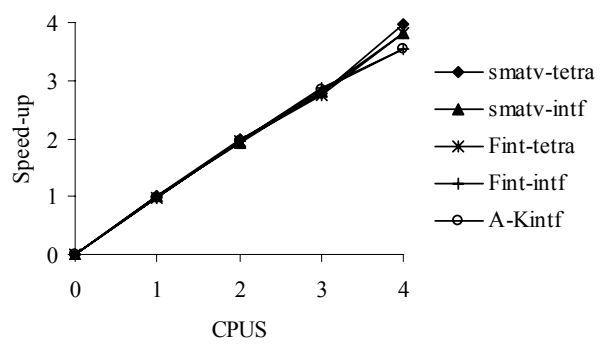

(b)

Fig. 10 (a) Program Summary of the ATEXPERT's Report

(b) ATEXPERT's Report -The top 6 subroutines on a Dedicated Speedup

According to ATEXPERT tool this program appears to be 99.2 percent parallel and 0.8 percent serial. Amdahl's Law predicts the program could expect to achieve a 3.9 times speedup on 4 cpus. A 3.8 speedup is predicted with 4 cpus on a dedicated system. 


\section{Concluding remarks}

This paper presented computational strategies used in an implementation of Rossi's probabilistic model for the simulation of cracking in concrete structures.

The resulting code achieved very good level, for parallel and vetor performance single CPU achieved $614 \mathrm{Mflop} / \mathrm{s}$ while parallel speed-up reached 3.8 in a 4 CPU system. The results emphasizes the suitability of the implemented code on a PVP system the CRAY T90

Extensive use of element-by-element techniques within the computational kernels comprised in the iterative solution drivers provided a natural way for achieving high flop rates and good parallel speed-up's.

The use of an adaptive inexact Newton method showed to be effective in reducing overall linear iterations, by a factor of two in comparison to the fixed tolerance inexact Newton scheme.

\section{Acknowledgments}

The authors are indebted to the Computer Graphics Technology Group TECGRAF/PUCRio, High Performance Computing Center NACAD/COPPE/UFRJ, and the Laboratory of Computational Methods in Engineering of the Program of Civil Engineering LAMCE /COPPE/UFRJ. CESUP/UFRGS is gratefully acknowledged for the computer time provided for the performance experiments.

This work was partially supported by CAPES and CNPq's grant $\mathrm{N}^{\circ} 150039 / 01-8(\mathrm{NV})$.

\section{References}

1. Rossi P., Richer S.: Numerical modeling of concrete cracking based on a stochastic approach. Materials and Structures, 20 (119), . (1987) 334-337.

2. Rossi P., Wu X., Maou F. le, and Belloc A.: Scale effect on concrete in tension. Materials and Structures, 27 (172), (1994) 437-444.

3. Rossi P., Ulm F.-J., and Hachi F.: Compressive behavior of concrete: physical mechanisms and modeling. Journal of Engineering Mechanics ASCE, 122 (11), (1996), 1038-1043.

4. Rossi P. and Ulm F.-J.: Size effects in the biaxial behavior of concrete: physical mechanisms and modeling. Materials and Structures, 30 (198), (1997) 210-216.

5. Paz C. N. M., Development and Implementation Probabilistic Model for 2D and 3D Discrete Cracking Concrete in Parallel Computing, D.Sc. Thesis, COPPE/UFRJ, the Graduate Institute of Federal University of Rio de Janeiro, Brazil (2000) [in Portuguese].

6. Press W. Teukolski H., S., Vetterling W.T. and Flannery B.: Numerical Recipes, Cambridge University Press (1992).

7. Fairbairn E. M. R., Paz C. N. M., Ebecken N. F. F., and Ulm F-J., Use of neural network for fitting of probabilistic scaling model parameter, Int. J. Fracture, 95, (1999), 315324.

8. Fairbairn E. M. R., Ebecken N. F. F., Paz C. N. M., and Ulm F-J.: Determination of probabilistic parameters of concrete: solving the inverse problem by using artificial neural networks, Computers and Structures, 78, (2000) 497-503. 
9. Kelley C. T.: Iterative Methods for Linear and Nonlinear Equations. Frontiers in applied mathematics, SIAM. Society for Industrial and Applied Mathematics, Philadelphia, (1995)

10. Hughes T. J. R., Ferenez R. M., Hallquist J. O.: Large-Scale Vectorized Implicit Calculation in Solid Mechanics on a CRAY X-MP/48 Utilizing EBE Preconditionated Conjugate Gradients Computer Methods in applied Mechanics and Engineering, 61, (1987) 2115-248.

11. Papadrakakis M.: Solving Large-Scale Problems in Mechanics: The Development and Application of Computational Solution, Editor, M. Papadrakakis, John Wiley and Sons, (1993).

12. Li Q. and Ansari F.: High Concrete in Uniaxial Tension, ACI Material J. 97- (1), (2000) 49- 57.

13. Coutinho A. L. G. A., Martins M. A. D., Alves J. L. D., Landau L., and Moraes A.: Edge-based finite element techniques for nonlinear solid mechanics problems, Int. J. for Numerical Methods in Engineering, 50 (9), (2001) 2050-2068.

14. Fairbairn E. M. R., Debeux V.J.C., Paz C. N. M., and Ebecken N. F. F.: Application of probabilistic Approach to the Analysis of gravity Dam Centrifuge Test, $8^{\text {th }}$ ASCE Specialty Conference on Probabilistic Mechanics and Structural Reliability (2000) PMC 2000-261.

15. Kelley C. T.: Iterative methods for optimization. Frontiers in applied mathematics, SIAM Society for Industrial and Applied Mathematics, Philadelphia, (1999). 